Psychological Medicine, 1992, 22, 551-555

Printed in Great Britain

\title{
EDITORIAL
}

\section{The role of macrophages in models of neurological and psychiatric disorder ${ }^{1}$}

We consider here some of the recent advances in knowledge about the possible functions of the macrophage in the normal and diseased or damaged nervous system. The macrophage forms the key element in interactions between the nervous system and the immune system. Macrophages have a truly remarkable life history. They are generated in the bone marrow, circulate in the blood as monocytes for a day or so and then take up residence in almost all tissues of the body, where they may reside for long periods, sometimes for many years. Tissue macrophages are highly specialized cells showing considerable heterogeneity from tissue to tissue. They play a role in tissue homeostasis but, possibly more importantly, they provide the first line of defence against injury and infection (Gordon, 1986). Tissue macrophages have only a limited capacity for proliferation, but their numbers may be rapidly augmented by recruitment of cells from the blood. These inflammatory or exudate macrophages have the potential to secrete a veritable catalogue of products, some of which are critical for tissue repair and others which may cause autologous tissue damage (Nathan, 1987).

Microglia were first described in the central nervous system (CNS) by Rio Hortega (1932), and from that time until the early 1980 s a controversy continued as to whether these cells were bloodderived cells or a type of neuroglia (see Ling, 1981 for review). In the last decade overwhelming evidence has accumulated that microglia are indeed the resident macrophages of the CNS (reviewed in Perry \& Gordon, 1991). In radiation chimeras cells bearing markers of donor leucocytes have been shown to enter the brain and adopt the form of microglia (Hickey \& Kimura, 1988). In the developing nervous system of rodents it has been shown, using antibodies to a macrophage-specific antigen, that monocytes enter the brain and pass through a series of morphological transitions to become microglia (Perry et al. 1985). With improvements in reagents and immunocytochemical methods there is now a long list of cell-surface and cytoplasmic antigens associated with microglia that are found on other leucocytes or restricted to macrophages (Streit et al. 1988; Perry \& Gordon, 1991). In addition to microglia within the parenchyma of the brain there are other specialized populations of macrophages associated with the microvasculature (Graeber et al. 1989), the choroid plexus and leptomeninges (Perry et al. 1985).

\section{MICROGLIA FUNCTION}

The functions of microglia are obscure. In the developing nervous system macrophages are observed phagocytosing cells dying as a consequence of naturally occurring cell death (Perry et al. 1985). Natural cell death is a major component of normal brain development, and up to $50 \%$ or more of the cells generated in some brain structures may degenerate during development (Oppenheim, 1981). There is evidence that macrophages secrete factors that may promote angiogenesis and gliogenesis in the developing CNS (Guillian et al. 1986, 1988). Whether there are any abnormalities in natural cell death or failures of macrophage phagocytosis of dying cells which have neurological consequences is at present unclear.

In the normal adult CNS microglia are ubiquitous but heterogeneous in their distribution and morphology (Perry et al. 1985; Lawson et al. 1990). The variations in density do not correlate with the amount of developmental cell death or with the distribution of any other known cell type or

' Address for correspondence: Dr V. Hugh Perry, Department of Pharmacology, Mansfield Road, Oxford OX13QT. 
neurotransmitter. A well-recognized feature of the microglia is their apparently quiescent nature when compared to other tissue macrophages (see Perry \& Gordon, 1991). Microglia lack a number of macrophage antigens, a fact which served to fuel the debate as to their relationship with macrophages. The ultrastructure of microglia also suggests a severely down-regulated phenotype; they have sparse Golgi apparatus and rough endoplasmic reticulum. Thus the CNS microenvironment produces a dramatic alteration in the morphology and phenotype of a macrophage. One factor that is involved is the blood-brain barrier (Perry \& Gordon, 1987). The barrier excludes large proteins from the CNS parenchyma, but in regions where the endothelium is fenestrated and cells are exposed to plasma proteins - the circumventricular organs - microglia retain several cellsurface antigens lacking on microglia isolated by the blood-brain barrier.

In one region of the normal adult CNS it has been shown that microglia retain their phagocytic capacity. In the posterior pituitary, where the axons of the magnocellular neurons terminate and secrete the hormones; oxytocin and vasopressin, microglia have been shown phagocytosing the endings of these healthy neurons (Pow et al. 1989). Whether this modelling of nerve endings is a constitutive function in other regions of the CNS is not known, but ultrastructural studies show microglia containing debris, particularly in older animals (Vaughn \& Peters, 1974).

\section{MICROGLIA IN NEUROPATHOLOGY}

It was well recognized by del Rio Hortega (1932) that microglia respond to many different forms of nervous system injury. The response that he documented was an increase in their numbers and alterations in their morphology. It has been suggested that there is probably no CNS pathological condition in the immunocompetent human in which a microglial reaction is absent (Duchen, 1984). With new antibodies available for the routine detection of microglia it is now possible to examine in further detail the microglial response in various neuropathological states. For example, microglia show morphological changes and enhanced expression of several surface antigens including major histocompatibility (MHC) Class II in Alzheimer's disease (McGeer et al. 1987, 1988; Akiyama \& McGeer, 1990), and Parkinson's disease (McGeer et al. 1988). In the putative autoimmune disease, multiple sclerosis, enhanced levels of MHC Class II antigens are seen on microglia (Hayes et al. 1987).

How do we interpret the microglial changes seen in these diseases? We know that other tissue macrophages will rapidly respond to tissue destruction and degeneration products, and thus a microglial response is just what we might expect of any tissue macrophage. It is possible that in chronic neurodegenerative diseases activated microglia might play a part in exacerbating the disease by the secretion of compounds that are neurotoxic. It is now clear that in HIV-1 infection of the CNS it is predominantly the cells of the mononuclear phagocytic lineage that are infected, and particularly the microglia (Vaseux et al. 1987; Kure et al. 1990). In the absence of HIV-1 infection of neurons it seems possible that the CNS dysfunction seen in acquired immunodeficiency syndrome (AIDS) is related to abnormalities of microglial function. Recent evidence from in vitro studies shows that HIV-1-infected monocytoid cell lines secrete compounds that are neurotoxic (Giulian et al. 1990). On the other hand, we know that macrophages play an important role in tissue repair processes (Leibovich \& Ross, 1975), and thus the microglial response may be important in repair processes during the disease.

\section{ANIMAL MODELS}

Insights into the possible role of microglia in pathological disorders of the nervous system can be gained from animal models. Unfortunately, in species such as rats and mice for which we have the best range of reagents for studying macrophages and microglia there are no directly comparable diseases.

Traumatic injury to the CNS provokes a clear microglial response, with alterations in the morphology and antigenic phenotypes of microglia and also recruitment of macrophages. These 
macrophages-microglia not only remove the debris, but their secretory products, such as interleukin-1, induce astrogliosis (Giulian et al. 1988), which may be important in re-establishing the blood-brain barrier. One striking feature that has emerged from studies of CNS traumatic injury is that the macrophage-microglial response to Wallerian degeneration in the CNS is slow, and the debris persists for a considerable period of time when compared to the peripheral nervous system (PNS) (Perry et al. 1987; Stoll et al. 1989a,b). While it may seem a rather academic question as to whether the debris is, or is not, removed from a fibre tract with little capacity for regeneration, recent evidence shows that CNS myelin contains proteins which can inhibit axon elongation and neuronal regeneration (Caroni \& Schwab, 1988; Schnell \& Schwab, 1990). Removal of the myelin debris may therefore be of some importance.

Microglia also respond around the cell body of an axotomized neuron. After injury to a peripheral nerve the microglia around the motoneurones in the spinal cord interpose their processes between the afferent synaptic boutons and the postsynaptic membrane-so-called synaptic stripping (Blinzinger \& Kreutzberg, 1968). The significance of synaptic stripping for protecting the neuron from degeneration is not clear, but it is of interest that in neuron populations, such as thalamic neurons, where synaptic stripping does not happen the neurons degenerate very rapidly (Matthews, 1973).

Although there are no diseases in rodents which match the human chronic neurodegenerative diseases, lesions induced by excitatory neurotoxins have been suggested to have some parallels. These compounds, which bind to glutamate receptors, cause prolonged depolarization and result in neuronal degeneration by a calcium-dependent mechanism. For example, injection of kainate or quinolinate into the striatum produces many of the morphological features of Huntington's disease (see DiFiglia, 1990). Intracerebral injection or epidural application of excitotoxins results in rapid neuronal degeneration and a vigorous macrophage-microglia response; the microglia appear morphologically activated and express new antigens including MHC Class II (Akiyama et al. 1988; Coffey et al. 1990). An interesting feature of the leucocyte response to this rapid cell death is that unlike acute destruction in other tissues there is essentially no response by the polymorphonuclear cells, and the macrophage-microglia numbers do not change for the first 48 hours after the lesion but then increase on the third day (Andersson et al. 1991). This suggests that there is some mechanism in the CNS that has evolved to 'short circuit' part of the inflammatory response, in particular the recruitment of polymorphonuclear cells, which may cause unwanted tissue damage and alterations in the blood-brain barrier.

The dramatic microglial response to excitatory neurotoxins led us to ask whether the prolonged excitatory activity such as that seen in epilepsy might produce alterations in the microglia. Injection of tetanus toxin produces limbic seizures in the rat (Mellanby et al. 1984). In this model of epilepsy microglia appear activated in both animals with cell loss and those with no apparent cell loss (Shaw et al. 1990). Thus, microglia may be activated by alterations in their local ionic environment, and it remains an important question as to whether they play a part in recovery from the seizures or may be involved in precipitating neuronal death.

Experimental allergic encephalitis (EAE) is a widely studied animal model of autoimmune disease. The disease is evoked by immunizing animals with a preparation of CNS myelin and adjuvant or by the transfer of sensitized T-lymphocytes. Damage to neuronal tissue is caused when sensitized T-lymphocytes recognize CNS antigens in collaboration with cells expressing MHC Class II antigens. This has provoked considerable interest as to which population of endogenous cells within the CNS express MHC Class II and under what conditions (see above). In EAE it is found that the only endogenous cells expressing MHC Class II antigens are the microglia (Vass et al. 1986). Enhancing the expression of MHC Class II antigen on microglia along the optic pathway by optic nerve section prior to the induction of EAE specifically enhances the leucocyte attack along the optic pathway (Konno et al. 1990). If the macrophage population is depleted, by the ingenious delivery of a cytotoxin to the macrophages, at the time that the disease would normally begin, disease can be dramatically reduced (Huitinga et al. 1990). Thus the resident cells may play a critical role in the induction of the disease and the recruited cells play a critical role in its evolution. 


\section{COMPARISON OF PNS AND CNS}

Nerve fibres of the CNS fail to regenerate, but fibres of the PNS retain this capacity. Do macrophages play a role in this repair process? Macrophages are rapidly recruited to the distal segment of an injured peripheral nerve; they phagocytose the debris and may also play a part in initiating Schwann cell division (Perry et al. 1987; Stoll et al. 1989a,b). In a remarkable mutant mouse in which peripheral nerves undergo very slow Wallerian degeneration and monocytes are not recruited (Lunn et al. 1989), the absence of Wallerian degeneration and the lack of macrophage recruitment does not impede regeneration of motor fibres (Lunn et al. 1989). However, sensory nerve fibres do not grow well down this undegenerated territory (Brown et al. 1991). A possible reason for this poor regeneration is that sensory fibres may require nerve growth factor, which is produced in the distal segment during normal Wallerian degeneration (Heumann et al. 1987). The failure of monocyte recruitment in this mutant mouse results in a paucity of nerve growth factor production (Brown et al. 1991). It appears that recruitment of monocytes is an important part of the regeneration process and may also potentiate the rate of Wallerian degeneration (Lunn et al. 1989).

\section{CONCLUSION}

Our knowledge of the functions of macrophages in the normal and diseased or disturbed nervous system is in its infancy. Macrophages play a part in the development of the CNS and then differentiate to form microglia. Microglia are a highly specialized form of tissue macrophage that appears to be down-regulated by the CNS microenvironment; this in turn may contribute to the modified inflammatory response seen in the CNS. Microglia are exquisite indicators of neuropathology in the adult CNS.

Studies in the PNS suggest that macrophages are an important component of both degeneration and regeneration of neurons. The emerging parallels between macrophage functions in the nervous system and other tissues suggest that future studies will further highlight the role of macrophages in neurological and psychiatric disorders.

V. HUGH PERRY

\section{REFERENCES}

Akiyama, H. \& McGeer, P. L. (1990). Brain microglia constitutively express $\beta-2$ integrins. Journal of Neuroimmunology 30, 81-93.

Akiyama, H., Itagaki, S. \& McGeer, P. L. (1988). Major histocompatibility antigen expression on rat microglia following epidural kainic acid lesions. Journal of Neuroscience Research 20 , 147-157.

Andersson, P.-B., Perry, V. H. \& Gordon, S. (1991). The kinetics and morphological characteristics of the macrophage-microglial response to kainic acid-induced neuronal degeneration. Neuroscience 42, 201-214.

Blinzinger, K. \& Kreutzberg, G. W. (1968). Displacement of synaptic terminals from regenerating motoneurons by microglia. Zeitschrift für Zellforschung Mikroskopie und Anatomie 85, 145-157.

Brown, M. C., Perry, V. H., Lunn, E. R., Gordon, S. \& Heumann, R. (1991). Macrophage dependence of peripheral sensory nerve regeneration: possible involvement of nerve growth factor. Neuron 6, 359-370.

Caroni, P. \& Schwab, M. E. (1988). Two membrane protein fractions from rat central myelin with inhibitory properties for neurite growth and fibroblast spreading. Journal of Cell Biology 106, 1281-1288.

Coffey, P. J., Perry, V. H. \& Rawlins, J. N. P. (1990). An investigation into the early stages of the inflammatory response following ibotenic acid-induced neuronal degeneration. Neuroscience 35, 121-132.
DiFiglia, M. (1990). Excitotoxic injury of the neostriatum: a model for Huntington's disease. Trends in Neuroscience 13, 286-289.

Duchen, L. W. (1984). General pathology of neurons and neuroglia. In Greenfield's Neuropathology, 4th edn. (ed. J. H. Adams, J. A. N. Corsellis and L. W. Duchen), p. 41. Arnold: London.

Gordon, S. (1986). Biology of the macrophage. Journal of Cell Science (suppl. 4), 267-286.

Graeber, M. B., Streit, W. J. \& Kreutzberg, G. W. (1989). Identity of ED2-positive perivascular cells in rat brain. Journal of Neuroscience Research 22, 103-106.

Guilian, D, Allen, R. L., Baker, T. J. \& Tomozawa, Y. (1986). Brain peptides and glial growth. I. Glia promoting factors as regulators of gliogenesis in the developing and injured central nervous system. Journal of Biology 102, 803-820.

Guilian, D., Woodward, J., Young, D. G., Krebs, J. F. \& Lachman, L. B. (1988). Interleukin- 1 injected into mammalian brain stimulates astrogliosis and neovascularization. Journal of Neuroscience 8, 2485-2490.

Guilian, D., Vaca, K. \& Noonan, C. A. (1990). Secretion of neurotoxins by mononuclear phagocytes infected with HIV-1. Science 250, 1593-1596.

Hayes, G. M., Woodroofe, M. N. \& Cuzner, M. L. (1987). Microglia are the major cell type expressing $\mathrm{MHC}$ Class II in human white matter. Journal of Neurological Science 80, 25-37.

Heumann, R., Korsching, S., Brantlow, C. \& Thoenen, H. (1987). Changes in nerve growth factor synthesis in non-neuronal cells in 
response to sciatic nerve transection. Journal of Cell Biology 104, $1623-1631$

Hickey, W. F. \& Kimura, H. (1988). Perivascular microglial cells of the CNS are bone marrow derived and present antigen in vivo. Science 239, 290-292.

Huitinga, I., van Rooijen, N., de Groot, C. J. A., Uitdehaag, B. M. J. \& Dijkstra, C. D. (1990). Suppression of experimental allergic encephalomyelitis in Lewis rats after elimination of macrophages. Journal of Experimental Medicine 172, 1025-1033.

Konno, H., Yamamoto, T., Suzuki, H., Yamamoto, H., Iwasaki, Y., Ohara, Y., Teranuma, H. \& Harata, N. (1990). Targeting of adoptively transferred experimental allergic encephalitis lesion at the sites of Wallerian degeneration. Acta Neuropathologica 80, 521-526.

Kure, K., Lyman, W. D., Weidenheim, K. M. \& Dickson, D. W. (1990). Cellular localization of an HIV-1 antigen in subacute AIDS encephalitis using an improved double-labeling immunohistochemical method. American Journal of Pathology 136, 1085-1092.

Lawson, L. J., Perry, V. H., Dri, P. \& Gordon, S. (1990). Heterogeneity in the distribution and morphology of microglia in the normal and adult mouse brain. Neuroscience 39, 151-170.

Leibovich, S. J. \& Ross, R. (1975). The role of the macrophage in wound repair. American Journal of Pathology 8, 71-91.

Ling, E. A. (1981). The origin and nature of microglia. Advances in Cellular Neurobiology 2, 33-82.

Lunn, E. R., Perry, V. H., Brown, M. C. Rosen, H. \& Gordon, S (1989). Absence of Wallerian degeneration does not hinder regeneration in peripheral nerve. European Journal of Neuroscience 1, 27-33.

McGeer, P. L., Itagaki, S., Tago, H. \& McGeer, E. G. (1987). Reactive microglia in patients with senile dementia of the Alzheimer type are positive for the histocompatibility glycoprotein HLA-DR. Neuroscience Letters 79, 195-200.

McGeer, P. L. Itagaki, S., Boyes, B. E. \& McGeer, E. G. (1988). Reactive microglia are positive for HLA-DR in the substantia nigra of Parkinson's and Alzheimer's disease brains. Neurology 38, 1285-1291.

Matthews, M. A. (1973). Death of the central neuron: an electron microscopic study of thalamic retrograde degeneration following cortical ablation, Journal of Neurocytology 2, 265-288.

Mellanby, J., Hawkins, C. A., Mellanby, H., Rawlins, J. N. P. \& Impey, M. E. (1984). Tetanus toxin as a tool for studying epilepsy. Journal of Physiologie (Paris) 79, 207-215.

Nathan, C. F. (1987). Secretory products of macrophages. Journal of Clinical Investigation 79, 319-326.

Oppenheim, R. W. (1981). Neuronal cell death and some related regressive phenomena during neurogenesis: a selective historical review and progress report. In Studies in Developmental Neurobiology: Essays in Honor of Victor Hamburger (ed. W. M. Cowan), pp. 74-133. Oxford University Press: Oxford.
Perry, V. H. \& Gordon, S. (1987), Modulation of CD4 on macrophages and microglia in rat brain. Journal of Experimental Medicine 166, 1138-1143.

Perry, V. H. \& Gordon, S. (1991). Macrophages and the nervous system. International Review of Cytology 125, 203-244.

Perry, V. H., Hume, D. A. \& Gordon, S. (1985). Immunohistochemical localization of macrophages and microglia in the adult and developing mouse brain. Neuroscience 15, 313-326.

Perry, V. H., Brown, M. C. \& Gordon, S. (1987). The macrophage response to central and peripheral nerve injury: a possible role for macrophages in regeneration. Journal of Experimental Medicine $165,1218-1223$.

Pow, D. V., Perry, V. H., Morris, J. F. \& Gordon, S. (1989). Microglia in the neurohypophysis associate with and endocytose terminal portions of neurosecretory neurons. Neuroscience 33, $567-578$.

del Rio Hortega, P. (1932). Microglia. In Cytology and Cellular Pathology of the Nervous System (ed. W. Penfield), pp. 482-534. Paul B. Hoeber: New York.

Schnell, L. \& Schwab, M. E. (1990). Axonal regeneration in the rat spinal cord produced by an antibody against myelin-associated neurite growth inhibitors. Nature 343, 269-272.

Shaw, J. A. G., Perry, V. H. \& Mellanby, J. (1990). Tetanus toxininduced seizures cause microglial activation in rat hippocampus. Neuroscience Letters 120, 66-99.

Streit, W. J. Graeber, M. B. \& Kreutzberg, G. W. (1988). Functional plasticity of microglia: a review. Glia 1, 301-307.

Stoll, G., Trapp, B. D. \& Griffin, J. W. (1989a). Macrophage function during Wallerian degeneration of rat optic nerve: clearance of degenerating myelin and la expression. Journal of Neuroscience 9, 2327-2335.

Stoll, G., Griffin, J. W., Li, C. W. \& Trapp, B. D. (1989b). Wallerian degeneration in the peripheral nervous system: participation of both Schwann cells and macrophages in myelin degeneration. Journal of Neurocytology 18, 671-683.

Vaseux, R., Brousse, N., Jarry, A., Hehin, D., Marche, C., Vendrenne, C., Mikol, J., Wolff, M., Michon, C., Rosenbaum, W., Bureau, J.-F., Montagnier, L. \& Brahic, M. (1987). AIDS subacute encephalitis. Identification of infected cells. American Journal of Pathology 126, 403-410.

Vass, K., Lassmann, H., Wekerle, H. \& Wisniewski, H. M. (1986). The distribution of Ia antigens in the lesions of rat acute experimental allergic encephalomyelitis. Acta Neuropathologica 70, 149-160.

Vaughn, J. E. \& Peters, A. (1974). Neuroglial cells in the cerebral cortex of rats from young adulthood to old age: an electron microscopic study. Journal of Neurocytology 3, 405-429. 